\title{
ASSOCIAÇÃO ENTRE VITAMINA D, CONTROLE GLICÊMICO E COMPLICAÇÕES MICROVASCULARES NO DIABETES TIPO 1
}

\author{
Association between vitamin D, glycemic control and microvascular complications in type 1 \\ diabetes
}

\section{Asociación entre la vitamina $D$, el control glicémico y las complicaciones microvasculares del Diabetes tipo I}

\author{
Camila Lousada Herbster Ferraz \\ Universidade Federal do Ceará - UFC - Fortaleza (CE) - Brasil \\ Hospital Universitário Walter Cantídio - HUWC - Fortaleza (CE) - Brasil
}

\author{
Angela Delmira Nunes Mendes \\ Hospital Universitário Walter Cantídio - HUWC - Fortaleza (CE) - Brasil \\ Universidade Federal do Ceará - UFC - Fortaleza (CE) - Brasil
}

Tania Maria Bulcão Lousada Ferraz

Hospital Geral de Fortaleza - HGF - Fortaleza (CE) - Brasil

Carlos Antonio Bruno da Silva

Universidade de Fortaleza - UNIFOR - Fortaleza (CE) - Brasil

\section{RESUMO}

Objetivo: Avaliar a associação entre os níveis de vitamina $\mathrm{D}$, os parâmetros do controle metabólico e a presença de complicações microvasculares em pacientes portadores de diabetes mellitus tipo1 (T1DM). Métodos: Estudo transversal, analítico e observacional, realizado em 2016, em Fortaleza, Ceará, com prontuários de cinquenta pacientes. Investigaram-se os dados clínicos e epidemiológicos: sexo, idade, IMC (índice de massa corporal), cor da pele, controle glicêmico, duração do diabetes, dose diária de insulina, presença de complicações microvasculares e dosagem de vitamina D por quimiluminescência. Utilizaram-se os testes exato de Fisher, $t$-Student e Mann-Whitney com p $<0,05$. Resultados: Observou-se deficiência de vitamina $\mathrm{D}$ em 34 (68\%) pacientes, com média de $25(\mathrm{OH})$ de vitamina $\mathrm{D}, 23,24 \pm 4,29 \mathrm{ng} / \mathrm{mL}$ no grupo vitamina D deficiente e $38,22 \pm 7,72 \mathrm{ng} / \mathrm{mL}$ no grupo vitamina D suficiente. Além disso, 37 pacientes $(78 \%)$ apresentaram hemoglobina glicada acima de 7\% e semelhante nos dois grupos. A dose diária de insulina no grupo vitamina $\mathrm{D}$ deficiente foi maior que no grupo vitamina $\mathrm{D}$ suficiente, $54,81 \pm 27,4$ vs 55,55 \pm 19,2, mas sem associação significativa com níveis de vitamina $\mathrm{D}$. O nível sérico da vitamina $\mathrm{D}$ não se associou com parâmetros clínicos e epidemiológicos, como: sexo, idade, IMC, cor da pele, controle glicêmico, duração do diabetes, dose diária de insulina e presença de complicações microvasculares. Conclusão: A deficiência de vitamina D esteve presente na maioria dos pacientes com T1DM avaliados. No entanto, sem associação entre essa deficiência e as variáves clínicas e epidemiológicas analisadas.

Descritores: Diabetes Mellitus; Vitamina D; Registros Médicos.

\section{ABSTRACT}

Objective: To assess the association between vitamin D levels, parameters of metabolic control and presence of microvascular complications in type 1 diabetes mellitus (TIDM) patients. Methods: Analytical and observational cross-sectional study of medical records of fifty patients carried out in 2016 in Fortaleza, Ceará. Clinical and epidemiological data were analyzed: sex, age, BMI (body mass index), skin color, glycemic control, duration of diabetes, daily insulin dose, presence of microvascular complications, and vitamin D assay by chemiluminescence. Fisher's test, students's $t$ test and Mann-Whitney U test were used with $p<0.05$. Results: Vitamin D deficiency was seen in 34 (68\%) patients, with a mean of $25(\mathrm{OH})$ vitamin D of $23.24 \pm 4.29 \mathrm{ng} / \mathrm{mL}$ in the Vitamin D deficiency group and $38.22 \pm 7.72 \mathrm{ng} / \mathrm{mL}$ in the normal Vitamin D group. In addition, 37 patients (78\%) exhibited glycated hemoglobin above 7\%, which was similar in booth groups. The daily insulin dose in the vitamin $D$ deficiency group was higher than in the normal vitamin D group, $54.81 \pm 27.4$ vs $55.55 \pm 19.2$, but with no significant association with vitamin $D$ levels. Vitamin D levels were not associated with clinical and epidemiological such as: sex, age, BMI, skin color, glycemic control, duration of diabetes, daily insulin dose and presence of microvascular complications or insulin daily dose. Conclusion: Vitamin D deficiency was present in most of the TIDM patients analyzed. However, such deficiency was not associated with the clinical and epidemiological variables analyzed.

Descriptors: Diabetes Mellitus; Vitamin D; Medical Records.

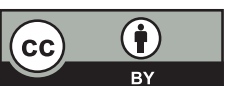

Este artigo está publicado em acesso aberto (Open Access) sob a licença Creative Commons, que permite uso, distribuição e reprodução em qualquer meio, sem restrições, desde que o trabalho seja corretamente citado.
Recebido em: 15/09/2017 Revisado em: $21 / 12 / 2017$

Aceito em: 25/04/2018 


\section{RESUMEN}

Objetivo: Evaluar la asociación entre los niveles de vitamina $D$, los parámetros del control metabólico y la presencia de complicaciones microvasculares de pacientes con Diabetes Mellitus Tipo I (DM1). Métodos: Estudio transversal, analítico y observacional realizado en 2016 en Fortaleza, Ceará, con historiales clínicos de cincuenta pacientes. Se investigaron los siguientes datos clínicos y epidemiológicos: el sexo, la edad, el IMC (Índice de Masa Corporal), el color de la piel, el control glicémico, la duración del diabetes, la dosis diaria de insulina, la presencia de complicaciones microvasculares y la dosificación de vitamina D por quimioluminiscencia. Se utilizaron las pruebas ecxato de Fisher, t-Student y Mann-Whitney con p<0,05. Resultados: Se observó la deficiencia de vitamina D en 34 (68\%) pacientes con media de 25(OH) de vitamina $D, 23,24 \pm 4,29 \mathrm{ng} / \mathrm{mL}$ en el grupo de vitamina $D$ deficiente y 38,22 7,72 $\mathrm{ng} / \mathrm{mL}$ en el grupo de vitamina D suficiente. Además, 37 pacientes (78\%) presentaron hemoglobina glicosilada por encima del 7\% y semejante para los dos grupos. La dosis diaria de insulina en el grupo de vitamina $D$ deficiente ha sido mayor que en el grupo de vitamina D suficiente, $54,81+27,4$ vs 55,55 + 19,2, pero sin asociación significativa con los niveles de vitamina $D$. El nivel sérico de vitamina $D$ no se ha asociado con los parámetros clínicos y epidemiológicos como el sexo, la edad, el IMC, el color de la piel, el control glicémico, la duración del diabetes, la dosis diaria de insulina y la presencia de complicaciones microvasculares. Conclusión: La deficiencia de vitamina D se dio en la mayoría de los pacientes con DM1 evaluados. Sin embargo, no hubo asociación entre la deficiencia y las variables clínicas y epidemiológicas analizadas.

Descriptores: Diabetes Mellitus; Vitamina D; Registros Médicos.

\section{INTRODUÇÃO}

A diabetes mellitus (DM) representa um grupo de doenças metabólicas, com etiologias diversas, caracterizado por hiperglicemia resultante de defeitos na secreção e/ou na ação da insulina ${ }^{(1)}$. A diabetes mellitus tipo 1 (T1DM) corresponde a 5 a $10 \%$ dos casos de diabetes, resultando, primariamente, da destruição celular autoimune das células $\beta$ pancreáticas associada com alteração na imunidade celular, humoral e predisposição genética ${ }^{(2-4)}$.

A incidência de T1DM tem aumentado cerca de 3\% ao ano ${ }^{(5)}$. No Brasil, essa incidência é em torno de 7,6/100 000 indivíduos com menos de 15 anos de idade ${ }^{(2)}$. Fatores ambientais parecem influenciar na epidemiologia do T1DM, como: dieta na infância e adolescência, nível de vitamina D, exposição solar, viroses, período de amamentação, desmame precoce e imunização ${ }^{(6)}$.

Estudos in vivo demonstraram que a 1,25 hidroxi $(\mathrm{OH})$ vitamina $\mathrm{D}$ inibe a expressão de citocinas inflamatórias, tais como: interleucina-1 $\beta$, interleucina-6, fator de necrose tumoral $\alpha$ (TNF- $\alpha$ ), interferon $\gamma$, interleucina-8 (IL 8) e interleucina 12 em indivíduos normais $^{(7)}$. A diabetes evidenciou a associação entre suplementação de $2000 \mathrm{UI}$ de vitamina D com redução do processo inflamatório e retardo na progressão da doença, com preservação de função de célula beta, mas sem repercussão no controle da glicemia ${ }^{(8)}$.

A suplementação de $25(\mathrm{OH})$ vitamina D durante a infância e a exposição materna durante a gravidez foram associadas a uma redução no risco de desenvolver T1DM. As crianças que tomaram 2000UI de vitamina D regularmente tinham um risco relativo (RR) de 0,22 (0,05-0,89), enquanto as crianças com suspeita de raquitismo durante o primeiro ano de vida tiveram RR de 3,0 $(1,0-9,0)$ para risco de $\mathrm{T}_{1} \mathrm{DM}^{(7)}$.

Crianças ou adultos jovens com T1DM, recém diagnosticados, exibem menores níveis de 25 (OH) vitamina D que os controles saudáveis ${ }^{(4,9)}$. Esses baixos níveis foram correlacionados com o aumento de biomarcadores de inflamação, incluindo proteína $\mathrm{C}$ reativa, e a expressão de receptores toll like (TLR) ${ }^{(10)}$.

Estudos demonstram uma relação causal entre deficiência de vitamina D e a presença de retinopatia em T1DM e T2DM. A fisiopatologia envolve a angiogênese e inflamação, causando danos nos vasos da retina ${ }^{(11-13)}$.

A insuficiência de vitamina $\mathrm{D}$ pode implicar na patogênese da albuminúria e a reposição de vitamina $\mathrm{D}$ reduz a proteinúria. No diabetes control and complications trial (DCCT), pacientes com níveis baixos de vitamina D foram associados com o maior risco de microalbuminúria, porém não foi demonstrada evidência em relação à perda da função renal e desenvolvimento de hipertensão ${ }^{(14)}$. Associação entre os níveis de vitamina D e a presença de neuropatia periférica em T1DM, além de exacerbação dos sintomas, também foram descritas em estudo anterior ${ }^{(15)}$.

No controle glicêmico, demonstra-se que a reposição de vitamina D em pacientes com T2DM ajuda na redução da hemoglobina glicada, porém o mesmo não ocorreu nos T1DM ${ }^{(16)}$. A associação do IMC com o status de vitamina D ainda é incerta e acreditase que a vitamina D regule a apoptose dos adipócitos, ocasionando a redução de massa gorda. No entanto, essa relação só foi observada em crianças com $\mathrm{T}_{2} \mathrm{DM}^{(16)}$.

A tireoidite de Hashimoto (TH) ocorre em 17 a $30 \%$ dos pacientes portadores de T1DM e cerca de $25 \%$ desses pacientes T1DM têm anticorpos tireoidianos, sugerindo a presença de autoimunidade na época do diagnóstico ${ }^{(1)}$. A ativação dos linfócitos T e B no T1DM pode inibir a expressão de antígenos tireoidianos levando a TH e a vitamina D teria um papel protetor nesse processo $^{(17)}$.

Em relação ao papel da vitamina D e o seu papel na promoção de saúde, um trabalho ${ }^{(18)}$ ressalta que ela tem sido um nutriente 
de interesse por conta de sua importância não apenas na saúde do osso como também na prevenção do câncer e da diabetes, no pleno funcionamento do sistema imunológico, neuropsicológico, bem como, em outras condições, como em processos inflamatórios e na doença cardiovascular ${ }^{(18)}$.

A partir das evidências das interrelações entre o controle da diabetes mellitus, o surgimento de complicações crônicas e os níveis de vitamina $\mathrm{D}$, este trabalho teve como objetivo avaliar a associação entre os níveis de vitamina $\mathrm{D}$, os parâmetros do controle metabólico e a presença de complicações microvasculares em pacientes portadores de diabetes mellitus tipo1 (T1DM).

\section{MÉTODOS}

Trata-se de um estudo transversal, analítico e observacional, realizado a partir de informações de prontuários de pacientes portadores de T1DM em acompanhamento no Ambulatório de Diabetes tipo 1 do Hospital Universitário Walter Cantídio da Universidade Federal do Ceará (HUWC/UFC), no período de 01 a 30 de janeiro de 2016. Selecionou-se a amostra por conveniência, sendo escolhidos os prontuários dos 50 primeiros pacientes atendidos no período da pesquisa.

As variáveis demográficas contemplavam: sexo, idade, grau de escolaridade (segundo estabelecido pelas Diretrizes do Ministério da Educação e Cultura do Brasil), renda familiar (em salários mínimos) e cor da pele autorreferida. As variáveis clínicas foram: idade ao diagnóstico, tempo de doença, índice de massa corporal (IMC) avaliado segundo valores estabelecidos pela Organização Mundial de Saúde (OMS) ${ }^{(17)}$, presença de complicações microvasculares, dose diária total de insulina; e variáveis laboratoriais: níveis de vitamina D, níveis de hemoglobina glicada e anticorpo anti peroxidadse (TPO).

A dosagem de vitamina $\mathrm{D}$ ocorreu pelo método imunoensaio de micropartículas por quimiluminescência (Automação Architect i2000 SR - ABBOTT). Quanto ao nível de vitamina D, estratificaram-se os pacientes em 2 subgrupos: 1) Grupo vitamina D suficiente se níveis $\geq 30 \mathrm{ng} / \mathrm{ml}$ e grupo Vitamina D deficiente se níveis $<30 \mathrm{ng} / \mathrm{ml}^{(7)}$. Pela relevância da vitamina D em pacientes diabéticos, esta dosagem tornou-se rotina no serviço e seu resultado encontra-se no prontuário médico.

Avaliou-se a dosagem de hemoglobina glicada pelo método cromatografia líquida de alta performance (HPLC), sendo utilizado o valor de $7 \%$ como de corte para discriminar o bom e mal controle. $\mathrm{O}$ anticorpo anti TPO foi avaliado quanto à presença ou ausência. A pesquisa da associação entre doença autoimune de tireoide e diabetes melitus tipo 1 é a rotina no serviço, e os resultados encontram-se no prontuário médico.

Os dados do estudo foram tabulados no software Excel for Windows, versão 2007, da Microsoft e analisados através do SPSS, versão 20.0, Texas, USA. A estatística descritiva foi expressa através de frequência e percentagem para variáveis qualitativas e médias e desvio-padrão para variáveis contínuas. Utilizaram-se os testes estatísticos Shapiro-Wilks, para verificar a normalidade da distribuição das variáveis quantitativas; Levene, para o teste de igualdade de variâncias e o exato de Fisher, para análise de variáveis categóricas. Para análise dos grupos de acordo com o nível de vitamina $\mathrm{D}$, aplicou-se o teste $t$-Student para as variáveis que possuem distribuição normal, e o teste de Mann-Whitney para as variáveis cuja distribuição não é normal. Adotou-se o nível máximo de significância estatística para os testes de $5 \%(p<0,05)$. A variação usada foi erro padrão da média em todas as variáveis.

O estudo recebeu aprovação pelo Comitê de Ética em Pesquisa do Hospital Universitário Walter Cantídio (HUWC) da Universidade Federal do Ceará sob Parecer nº 1.383.656.

\section{RESULTADOS}

Analisaram-se os dados de 50 prontuários de pacientes com T1DM atendidos no ambulatório de diabetes do HUWC. Do total da amostra, 15 (30\%) eram do sexo masculino, com média da idade de 26,9 $\pm 11,13$ anos, sendo a idade mínima de 10 e a máxima de 50 anos, com mediana de 26 anos. Segundo o grau de escolaridade, 22 (44\%) tinham ensino médio e 15 (30\%), ensino fundamental. Em relação à cor da pele, 24 (48\%) pacientes eram da cor branca e $26(52 \%)$ eram de cor não branca. A renda familiar mais frequente foi de menos de 2 salários mínimos, encontrada em 22 (44\%) pacientes (Tabela I).

A média de idade ao diagnóstico e o tempo da doeça foram 13,90 $\pm 9,4$ anos e 12,83 $\pm 7,8$ anos, respectivamente. O IMC, em média, foi de $24,05 \pm 3,9$, com a mediana de 24,0 . Na avaliação das complicações microvasculares, a nefropatia e a neuropatia estavam presentes em $10(20 \%)$ pacientes, seguidas de retinopatia em $8(16 \%)$ (Tabela I). Quanto ao tratamento, diferentes esquemas de insulina eram utilizados pelos pacientes. A média de dose diária total de insulina foi 55,31 $\pm 21,8 \mathrm{UI} /$ dia (Tabela I).

De acordo com os dados de hemoglobina glicada, 39 (78\%) pacientes apresentaram valores $\geq 7 \%$, indicando um mau controle glicêmico. Com relação à presença de disfunção de tireoide, 10 (20\%) eram portadores de hipotiroidismo, sendo que, destes, 30 (60\%) tinham anticorpo anti - TPO positivo. Da amostra pesquisada, 16 (32\%) pacientes encontravam-se com níveis suficientes de vitamina D e 34 (68 \%) com níveis deficientes de vitamina D (Tabela I).

Os níveis de vitamina D não mostraram associação com as variáveis demográficas (IMC, sexo e cor da pele) nem com as complicações microvasculares (retinopatia e neuropatia). A nefropatia esteve presente em $8(23,5 \%)$ pacientes do grupo vitamina D deficiente e em 2 (12,5\%) pacientes do grupo vitamina D suficiente. Portanto, não houve diferença estatisticamente significativa $(\mathrm{p}=0,498)$. (Tabela I). 
Tabela I - Características descritivas da amostra de pacientes portadores de diabetes mellitus tipo 1 e associação com o nível plasmático de vitamina D. Fortaleza, Ceará, Brasil, 2016.

\begin{tabular}{|c|c|c|c|c|c|c|c|}
\hline \multirow[b]{3}{*}{ Características } & \multicolumn{4}{|c|}{ Nível de Vitamina D } & & & \multirow{3}{*}{$\mathbf{p}$} \\
\hline & \multicolumn{2}{|c|}{ Total } & \multicolumn{2}{|c|}{ Deficiente } & \multicolumn{2}{|c|}{ Suficiente } & \\
\hline & $\mathbf{n}$ & $\%$ & $n$ & $\%$ & $\mathrm{n}$ & $\%$ & \\
\hline \multicolumn{8}{|l|}{ Sexo } \\
\hline Masculino & 15 & 30 & 8 & $23,5 \%$ & 7 & $43,80 \%$ & 0,191 \\
\hline Feminino & 35 & 70 & 26 & $76,5 \%$ & 9 & $56,30 \%$ & \\
\hline \multicolumn{8}{|l|}{ Grau de escolaridade } \\
\hline Superior & 12 & 24 & 9 & $26,5 \%$ & 3 & $18,80 \%$ & 0,925 \\
\hline Ensino médio & 22 & 44 & 14 & $41,2 \%$ & 8 & $50,00 \%$ & \\
\hline Analfa/fundam & 16 & 32 & 11 & $32,4 \%$ & 5 & $31,30 \%$ & \\
\hline \multicolumn{8}{|l|}{ Renda familiar } \\
\hline$\leq 1 \mathrm{sm}$ & 24 & 48 & 16 & $47,1 \%$ & 8 & $50,00 \%$ & 1 \\
\hline$\geq 2 \mathrm{sm}$ & 26 & 52 & 18 & $52,9 \%$ & 8 & $50,00 \%$ & \\
\hline \multicolumn{8}{|l|}{ Cor da pele } \\
\hline Branca & 24 & 48 & 18 & $52,9 \%$ & 6 & $37,50 \%$ & 0,372 \\
\hline Nao branca & 26 & 52 & 16 & $47,1 \%$ & 10 & $62,50 \%$ & \\
\hline \multicolumn{8}{|l|}{ Comorbidades } \\
\hline Tireopatia & 10 & 20 & 9 & $26,5 \%$ & 1 & $6,30 \%$ & 0,138 \\
\hline Retinopatia & 8 & 16 & 5 & $14,7 \%$ & 3 & $18,80 \%$ & 0,699 \\
\hline Nefropatia & 10 & 20 & 8 & $23,5 \%$ & 2 & $12,50 \%$ & 0,498 \\
\hline Neuropatia & 10 & 20 & 7 & $20,6 \%$ & 3 & $18,80 \%$ & 1 \\
\hline Ac Anti TPO & 10 & 20 & 8 & $34,8 \%$ & 2 & $22,20 \%$ & 0,681 \\
\hline \multicolumn{8}{|l|}{ Tratamento } \\
\hline insulina & 40 & 80 & 27 & $79,4 \%$ & 13 & $81,30 \%$ & 1 \\
\hline Insulina $+\mathrm{AO}$ & 10 & 20 & 5 & $14,7 \%$ & 4 & $25,00 \%$ & 0,442 \\
\hline \multicolumn{8}{|l|}{ HbA1C } \\
\hline$<7 \%$ & 11 & 22 & 6 & $17,6 \%$ & 5 & $31,30 \%$ & 0,297 \\
\hline$\geq 7 \%$ & 39 & 78 & 28 & $82,4 \%$ & 11 & $68,80 \%$ & \\
\hline
\end{tabular}

Teste Exato de Fischer; Analfa/fundam: analfabetos ou ensino fundamental; AO: antidiabéticos orais; Ac Anti TPO: anticorpo antiperoxidase; HbA1C: hemoglobina glicada.

O grupo vitamina $\mathrm{D}$ suficiente apresentou níveis de vitamina $\mathrm{D}$, em média, de $38,22 \pm 7,7$ e o grupo vitamina $\mathrm{D}$ deficiente, em média, de 23,24 \pm 4,2 (Tabela II).

Ao ser analisada a associação das variáveis demográficas entre os grupos de acordo com o nível plasmático de vitamina $\mathrm{D}$, não houve diferença na média de idade. A idade ao receber o diagnóstico de diabetes também foi semelhante entre os grupos, sendo o tempo de diagnóstico em média de 13,9 anos $(14,9 v s$ 13,4, $\mathrm{p}=0,60)$. Contudo, a presença de tireopatia foi mais frequente em pacientes do grupo vitamina D deficiente, ou seja, $9(26,5 \%)$ pacientes, aumentando o poder para $10 \%$ da prevalência de tireopatia no grupo vitamina D deficiente. Foi significativamente maior que no grupo vitamina $\mathrm{D}$ suficiente $(\mathrm{p}=0,01)$ (Tabela II).

Não foi evidenciada associação entre níveis de hemoglobina glicada e os níveis de vitamina D nos pacientes T1DM estudados. A dose diária total de insulina utilizada no grupo vitamina D deficiente foi maior que no grupo vitamina $\mathrm{D}$ suficiente $(55,55 \pm$ 19,2 vs 54,81 $\pm 27,4$ ), mas não mostrou associação significativa com os níveis de vitamina D (Tabela II).

\section{DISCUSSÃO}

Estudos têm mostrado a associação entre a deficiência de vitamina $\mathrm{D}$ e a T1DM $\mathrm{DM}^{(7-9,12,19)}$. Um estudo finlandês, mostrou que, depois da suplementação de vitamina D, por 220 crianças finlandesas, a partir de 2003, a incidência de diabetes tipo 1 reduziu e atingiu um platô $\hat{0}^{(20)}$. 
Tabela II - Associação das variáveis contínuas clínicas e laboratoriais de acordo com o nível plasmático da vitamina D de pacientes diabéticos tipo 1. Fortaleza, Ceará, Brasil, 2016.

\begin{tabular}{|c|c|c|c|c|c|c|c|}
\hline \multirow[b]{3}{*}{ Variáveis } & \multicolumn{7}{|c|}{ Classificaçao vitamina D } \\
\hline & \multicolumn{3}{|c|}{ Deficiente (34) } & \multicolumn{3}{|c|}{ Suficiente (16) } & \multirow[b]{2}{*}{$\mathbf{p}$} \\
\hline & Média & DP & Mediana & Média & DP & Mediana & \\
\hline Idade (anos) & 26,2 & 11,07 & 24,5 & 28,4 & 11,48 & 28,0 & 0,52 \\
\hline Idade ao diagnóstico (anos) & 13,4 & 8,87 & 11 & 14,9 & 10,71 & 14,5 & 0,739 \\
\hline Tempo de doença (anos) & 12,6 & 8,36 & 11 & 13,4 & 6,83 & 12,5 & 0,479 \\
\hline IMC $\left(\mathrm{Kg} / \mathrm{m}^{2}\right)$ & 24,4 & 3,47 & 24 & 23,4 & 4,97 & 22,6 & 0,453 \\
\hline Hemoglobina glicada & 9,1 & 2,33 & 8,5 & 8,0 & 1,63 & 8,0 & 0,102 \\
\hline Dose diária insulina & 55,6 & 19,22 & 52,5 & 54,8 & 27,44 & 53,5 & 0,913 \\
\hline
\end{tabular}

Teste t-Student, teste de Mann-Whitney. DP: desvio padrão; IMC $\left(\mathrm{Kg} / \mathrm{m}^{2}\right)$ : índice de massa corporal (quilo por metro quadrado)

A prevalência de níveis baixos de vitamina D são comuns na população, tanto jovem como adulta, quer por baixa ingestão, baixa exposição solar, quer por distúrbios absortivos, e tem se tornado um problema de saúde pública ${ }^{(7,21,22)}$. Alta prevalência de níveis mais baixos de vitamina D em pacientes com T1DM também é descrita por outros autores ${ }^{(7,21-23)}$.

O presente estudo mostrou uma prevalência de $68 \%$ de pacientes diabéticos tipo 1 com deficiência de vitamina D. Em trabalho semelhante, a prevalência de pacientes vitamina D deficientes, entre os pacientes com T1DM, foi de 15\%; de insuficiente foi de $61 \%$ e suficiente, de $24 \%{ }^{(20)}$. Não houve diferença quanto ao sexo, cor da pele e IMC, concordante aos dados da atual pesquisa. Um estudo avaliando 30 crianças com T1DM demonstrou também elevada prevalência de deficiência de vitamina D, das quais $50 \%$ eram deficientes e $45 \%$ eram insuficientes ${ }^{(22)}$. Outra publicação, em que foram avaliados 60 adultos com T1DM na Arábia Saudita, $100 \%$ deles eram deficientes em vitamina $\mathrm{D}^{(23)}$. Trabalho anterior detectou deficiência de vitamina $\mathrm{D}$ em $94,4 \%$ de 72 pacientes com diabetes e 58,5\% dos 41 controles saudáveis. Além disso, a deficiencia grave de vitamina D foi mais presente nos pacientes T1DM $(60 \%)$ do que nos controle $(8,3 \%)^{(24)}$.

Uma metanálise publicada sobre estudos observacionais sugere que o risco de desenvolver T1DM é baixo em crianças que receberam suplemento de vitamina D em comparação com aquelas que não receberam (odds ratio $0,71,95 \%$ IC 0,60 a 0,84 ) e a suplementação entre 7 e 12 meses é mais benéfica entre o nascimento e os 06 meses $^{(25)}$.

$\mathrm{O}$ atual estudo não evidenciou associação dos níveis de vitamina D com faixa etária ou sexo, da mesma forma que outro trabalho $^{(24)}$ não mostrou diferença nos níveis de vitamina $\mathrm{D}$ entre crianças do sexo masculino e feminino diabéticos, apesar de na população não diabética ter sido observada uma maior prevalênciade deficiencia de vitamina $\mathrm{D}$ nas do sexo feminino $(65 \%$ vs $52,4 \%)^{(24)}$.

Apesar da deficiência de vitamina D ter associação com o risco aumentado de desenvolvimento de T1DM segundo estudos de metanálise ${ }^{(26,27)}$, há controvérsias no efeito da ação da vitamina D e controle glicêmico em T1DM. Estudos têm mostrado que pacientes que receberam suplementação e alcançaram níveis suficientes de vitamina D têm redução da hemoglobina glicada, associando-se à melhora no controle glicêmico. Por outro lado, outros estudos não demonstraram essa associação, concordante com os dados da presente pesquisa ${ }^{(16,28,29)}$.

Além disso, no atual estudo, não se encontrou associação entre a dose diária total de insulina utilizada pelos pacientes e os níveis de vitamina $\mathrm{D}$, similar ao estudo prévio realizado em uma população de T1DM em São Paulo ${ }^{(8)}$.

A severidade da retinopatia diabética foi inversamente relacionada com o nível de vitamina D em pacientes T2DM, mas poucos dados existem na literatura sobre essas complicações microvasculares em T1DM. Existe a associação entre deficiência de vitamina $\mathrm{D}$ e retinopatia diabética, independente da duração do diabetes e da hemoglobina glicada ${ }^{(11)}$. Receptores da vitamina D estão presentes na retina, e polimorfismos nesses receptores podem ser responsáveis pelo grau de severidade da retinopatia, provavelmente devido a efeito angiogênico da vitamina D. Este presente estudo não mostrou associação significativa entre deficiência de vitamina D com a presença de retinopatia, semelhante ao estudo dinamarquês desenvolvido no Steno Diabetes Center $^{(12)}$.

Dois grandes clássicos estudos em T1DM, como o DCCT (The Diabetes Control and Complication Trial) e EDIC (Epidemiology of Diabetes Interventions and Complications Study Research), não demonstraram associação entre baixos níveis de vitamina D e o risco de desenvolver nefropatia em pacientes com T1DM concordante com o estudo em questão ${ }^{(13)}$.

A positividade do Ac anti-TPO (anticorpo antiperoxidase), mais prevalente no grupo vitamina D deficiente (34,8\%) no atual estudo, sugere a falta de ação protetora da vitamina D no processo imunológico da tireoidite ${ }^{(19,30)}$.

Na perspectiva da saúde global, é geralmente aceito que a deficiência de vitamina D é um problema de saúde mundial que afeta não apenas a saúde músculo-esquelética, mas também está relacionada a um grande numero de doenças crônico-degenerativas, como o diabetes. No entanto, ainda permanece a falta de estudos e ensaios clínicos randomizados para apoiar a evidência desses benefícios para a saúde, não relacionada apenas ao esqueleto e metabolismo do cálcio ${ }^{(31)}$. 
As limitações do presente estudo dizem respeito ao tamanho da amostra, a ausência de dados do padrão de alimentação e de informações sobre suplementação prévia de vitamina D. Estudos posteriores deveriam ser realizados para sugerir dosagem de vitamina D em todos os pacientes portadores de DM1, com intuito de melhorar o controle glicêmico, os parâmetros metabólicos e retardar complicações microvasculares, elevando, assim, a saúde desses pacientes.

\section{CONCLUSÃO}

A deficiência de vitamina D esteve presente na maioria dos pacientes investigados com T1DM. No entanto, não foi encontrada associação entre essa deficiência e as variáves sexo, idade, IMC, controle glicêmico, tempo de diabetes, dose total diária de insulina e a presença de complicações microvasculares.

\section{REFERÊNCIAS}

1. American Diabetes Association. Diagnosis and classification of diabetes mellitus. Diabetes Care. 2017;40 (Suppl 1):S11-24

2. Milech A, Perez A, Golbert AA et al. Diretrizes da Sociedade Brasileira de Diabetes (2015-2016). São Paulo: A.C. Farmacêutica; 2016.

3. Shaw JE, Sicree RA, Zimmet PZ. Global estimates of the prevalence of diabetes for 2010 and 2030. Diabetes Res Clin Pract. 2010;87(1):4-14.

4. Wolden-Kirk H, Overbergh L, Christesen HT, Brusgaard K, Mathieu C. Vitamin D and diabetes: its importance for beta cell and immune function. Mol Cell Endocrinol. 2011;347(1-2):106-20.

5. Atkinson MA, Eisenbarth GS, Michels AW. Type 1 diabetes. Lancet, 2014;383(9911):69-82.

6. Egro FM. Why is type 1 diabetes increasing? J Mol Endocrinol. 2013;51(1):R1-13.

7. Griz LH, Bandeira F, Gabbay MA, Dib SA, Carvalho EF. Vitamin D and diabetes mellitus: an update 2013. Arq Bras Endocrinol Metabol. 2014;58(1):1-8.

8. Gabbay MA, Sato MN, Finazzo C, Duarte AJ, Dib AS. Effect of cholecalciferol as adjunctive therapy with insulin on protective immunologic profile and decline of residual $\beta$-cell function in new-onset type 1 diabetes mellitus. Arch Pediatr Adolesc Med. 2012;166(7):601-7.

9. Mishra A, Dayal D, Sachdeva N, Attri SV. Effect of 6-months' vitamin D supplementation on residual beta cell function in children with type 1 diabetes: a case control interventional study. J Pediatr Endocrinol Metab. 2016;29(4):395-400.

10. Devaraj S, Jian I, Yun JM, Bremer. A demonstration of increased toll-like receptor 2 and toll-like receptor 4 expression in monocytes of type 1 diabetes mellitus patients with microvascular complications. Metabolism. 2011;60(2):256-9.

11. Kaur H, Donaghue KC, Chan AK, Benitez-Aguirre P, Hing S, Lloyd M, et al. Vitamin D deficiency is associated with retinopathy in children and adolescents with type 1 diabetes. Diabetes Care. 2011;34(6):1400-2.

12. Joergensen C, Hovind P, Schmedes A, Parving HH, Rossing P. Vitamin D levels, microvascular complications, and mortality in type 1 diabetes. Diabetes Care. 2011;34(5):1081-5.

13. Boer IH, Sachs MC, Cleary PA, Hoofnagle AN, Lachin JM, Molitch ME, et al. Diabetes control and complication trial/ epidemiology of diabetes interventions and complications study research group: circulating vitamin d metabolites and kidney disease in type 1 diabetes. J Clin Endocrinol Metab. 2012;97(12):4780-8.

14. Shimo N, Yasuda T, Kaneto H, Katakami N, Kuroda A, Sakamoto F, et al. Vitamin D deficiency is significantly associated with retinopathy in young Japanese type 1 diabetic patients. Diabetes Res Clin Pract. 2014;106(2):e 41-3.

15. Alam U, Arul-Devah V, Javed S, Malik RA. vitamin d and diabetic complications: true or false prophet? Diabetes Ther. 2016;7(1):11-26.

16. Nwosu BU, Maranda L. The effects of vitamin D supplementation on hepatic dysfunction, vitamin D status, and glycemic control in children and adolescents with vitamin D deficiency and either type 1 or type 2 diabetes mellitus. PLoS One. 2014;9(6):e 99646.

17. World Health Organization. Obesity: preventing and managing the global epidemic. Report of a World Health Organization Consultation. Geneva: WHO; 2000. (WHO Obesity Technical Report Series, n. 284).

18. Cadenhead K. Council on health promotion: the vitamin D debate. BC Med J. 2015;57(9):414. 
19. Muscogiuri G, Mitri J, Mathieu C, Badenhoop K, Tamer G, Orio F, et al. Mechanisms in endocrinology: vitamin D as a potential contributor in endocrine health and disease; Eur J Endocrinol. 2014;171(3):R101-10.

20. Mäkinen MV, Mykkänen J, Ilonen J, Veijola R, Hyöty H, Knip M, et al. An increase in serum 25-hydroxyvitamin d concentrations preceded a plateau in type 1 diabetes incidence in finnish children. J Clin Endocrinol Metab. 2014;99(11):e2353-6.

21. Svoren BM, Volkening LK, Wood JR, Laffel LM. Significant vitamin D deficiency in youth with type 1 diabetes mellitus. J Pediatr. 2009;154(1):132-4.

22. Savastio S, Cadario F, Genoni G, Bellomo G, Bagnati M, Secco G, et al. Vitamin D Deficiency and Glycemic Status in Children and Adolescents with Type 1 Diabetes Mellitus. PLoS One. 2016;11(9):e0162554.

23. Al-Daghri NM, Al-Attas OS, Alokail MS, Alkharfy KM, Yakout SM, Aljohani NJ, et al. Lower vitamin D status is more common among Saudi adults with diabetes mellitus type 1 than in non-diabetics. BMC Public Health. 2014;14:153.

24. Daga RA, Laway BA, Shah ZA, Mir SA, Kotwal SK, Zargar AH. High prevalence of vitamin D deficiency among newly diagnosed youth-onset diabetes mellitus in north India. Arq Bras Endocrinol Metab. 2012;56(7):423-8.

25. Zipitis CS, Akobeng AK. Vitamin D supplementation in early childhood and risk of type 1 diabetes: a systematic review and meta-analysis. Arch Dis Child. 2008;93(6):512-7.

26. Raab,J, Giannopoulou EZ, Schneider S, Warncke K, Krasmann M, Winkler C, et al. Prevalence of vitamin D deficiency in pre-type 1 diabetes and its association with disease progression. Diabetologia. 2014;57(5):902-8.

27. Miettinen ME, Reinert L, Kinnunen L, Harjutsalo V, Koskela P, Surcel HM, et al. Serum 25-hydroxyvitamin D level during early pregnancy and type 1 diabetes risk in the offspring. Diabetologia. 2012;55(5):1291-4.

28. Al Sawah S, Compher CW, Hanlon AL, Lipman TH. 25-Hydroxyvitamin D and glycemic control: a cross-sectional study of children and adolescents with type 1 diabetes. Diabetes Res Clin Pract. 2016;115:54-9.

29. Vitamin D supplement in early childhood and risk for type I (insulin-dependent) diabetes mellitus. The EURODIAB Substudy 2 Study Group. Diabetologia. 1999;42(1):51-4.

30. Treiber G, Prietl B, Fröhlich-Reiterer E, Lechner E, Ribitsch A, Fritsch M, et al. Cholecalciferol supplementation improves suppressive capacity of regulatory T-cells in young patients with new-onset type 1 diabetes mellitus: a randomized clinical trial. Clin Immunol. 2015;161(2):217-24.

31. Hossein-nezhad A, Holick MF. Vitamin D for health: a global perspective. Mayo Clinic Proc. 2013;88(7):720-55.

\section{Endereço do primeiro autor:}

Camila Lousada Herbster Ferraz

Universidade Federal do Ceará - UFC

Rua Capitão Francisco Pedro, 1290

Bairro: Rodolfo Teófilo

CEP: 60430-372 - Fortaleza - CE - Brasil

E-mail: camilaherbster@hotmail.com

\section{Endereço para correspondência:}

Carlos Antonio Bruno da Silva

Universidade de Fortaleza - UNIFOR

Programa de Pós Graduação em Saúde Coletiva

Av. Washington Soares, 1321

Bairro: Edson Queiroz

CEP: 60811-905 - Fortaleza - CE - Brasil

E-mail: carlosbruno@unifor.br 